\title{
BMJ Open First trimester screening for pre- eclampsia and intrauterine growth restriction using three-dimensional Doppler angiography (SPIRIT): protocol for a multicentre prospective study in nulliparous pregnant women
}

Charline Bertholdt (D , ,2 Gabriela Hossu, ${ }^{2,3}$ Claire Banasiak, ${ }^{3}$ Marine Beaumont, ${ }^{2,3}$ Olivier Morel ${ }^{1,2}$

To cite: Bertholdt C, Hossu G, Banasiak C, et al. First trimester screening for pre-eclampsia and intrauterine growth restriction using three-dimensional Doppler angiography (SPIRIT): protocol for a multicentre prospective study in nulliparous pregnant women. BMJ Open 2020;10:e037751. doi:10.1136/ bmjopen-2020-037751

- Prepublication history and supplemental material for this paper are available online. To view these files, please visit the journal online (http://dx.doi. org/10.1136/bmjopen-2020037751).

Received 06 March 2020 Revised 13 August 2020 Accepted 18 August 2020
Check for updates

(c) Author(s) (or their employer(s)) 2020. Re-use permitted under CC BY-NC. No commercial re-use. See rights and permissions. Published by BMJ.

${ }^{1}$ Obstetric and Fetal Medicine Unit, CHRU Nancy, Nancy, France ${ }^{2}$ Inserm IADI, Université de Lorraine, Nancy, France ${ }^{3}$ Inserm CIC-IT, CHRU Nancy, Nancy, France

Correspondence to Dr Charline Bertholdt; charline.bertholdt@gmail.com

\section{ABSTRACT}

Introduction Pre-eclampsia (PE) and intrauterine growth restriction (IUGR) are two major pregnancy complications, related to chronic uteroplacental hypoperfusion.

Nowadays, there is no screening or diagnostic test for uteroplacental vascularisation deficiency in pregnant women. Since 2004, 3 three-imensional power Doppler (3DPD) angiography has been used for the evaluation of uteroplacental vascularisation and three vascular indices are usually calculated: Vascularisation Index (VI), Flow Index (FI) and vascularisation-FI (VFI). A high intraobserver and interobserver reproducibility and a potential interest for placental function study were reported by our team and others.

The main objective of our study is to determine differences in 3DPD indices at first trimester between pregnancies defined at their outcome as uncomplicated pregnancy, PE (mild and severe) and IUGR in nulliparous women.

Methods and analysis This is a national multicentre prospective cohort study conducted in four French maternity units. We expect to include 2200 women in a period of 36 months. The nulliparous pregnant women will be recruited during their first trimester consultation (11-13+6 gestation week (GW)). The 3DPD and uterine artery Doppler acquisition will be included in the current routine 11-13+6 GW ultrasound. Also, additional blood samples will be taken for biomarker analysis (PAPP-A and P1GF) and biological collection. Uteroplacental VIs (FI and VFI) will be measured. For each subgroup (uncomplicated pregnancy, PE and IUGR), mean values in 3DPD indices will be computed and compared using a pairwise t test with a Bonferroni correction $\mathrm{p}$ value adjustment.

Ethics and dissemination The study was approved by the French Ethics Committee, the Comité de Protection des Personnes SUD MEDITERRANEE IV on 13 February 2018 with reference number 171203 . The results of this study will be published in a peer-reviewed journal and will be presented at relevant conferences.

Trial registration number NCT03342014; Pre-results. PHRCN-16-0567.

\section{Strengths and limitations of this study}

This is a controlled study with a homogeneous population (thanks to strict inclusion criteria) and a standardised acquisition technique of three-dimensional power Doppler (3DPD).

- Different settings of 3DPD will be used and compared with evaluate their impact on measured indices (Vascularisation Index, Flow Index (FI) and Vascularisation FI).

- Reproducibility of the acquisitions will also be evaluated.

- This is the first large cohort study to evaluate the interest of 3DPD in both pre-eclampsia and intrauterine growth restriction.

- The volume of interest will be manually drawn in VOCAL software which will make the technique dependent on the operator.

\section{INTRODUCTION}

Pre-eclampsia (PE) and intrauterine growth restriction (IUGR) are the main complications of pregnancy in Europe. These pathologies, which affect $4 \%-7 \%$ of pregnancies, are considered to be related to a chronic uteroplacental hypoperfusion. ${ }^{1}$ Major advances in the uteroplacental physiological understanding were made by Donner and Ramsey in 20 th century. ${ }^{23}$

It is admitted that after implantation, trophoblast cells start to induce vascular remodelling, first begining in endometrium and then in adjacent myometrium, which can be detected by Doppler imaging. ${ }^{4-6}$ With these modifications, a rich and dense vascular network is developed in the myometrium under the placental basal plate. The development of this vascular architecture coincides 
with the terminal parts of spiral arteries changes induced by extravillous trophoblast cells invasion. An anatomical and functional arteriovenous shunt, which plays a major role in the gas exchange, was found to be developed in the subplacental myometrium from first trimester until the end of pregnancy by Schaaps and Tsatsaris, who took part in this research consortium (Port-Royal Maternity). ${ }^{7}$ The timing of perfusion of the intervillous chamber has yet to be further evaluated, and our research group is actually involved in such research. ${ }^{8}$

Because of the impossibility of using contrast agent enhanced imaging techniques during pregnancy, Doppler imaging remains the only way to assess the vascularisation. Up to 2004, only the principal afferent vascular flow, originating from uterine artery, could be evaluated. The impaired placental perfusion, which is associated with development of PE/IUGR could be reflected in an increased uterine artery Pulsatility Index (PI). With uterine artery PI, the detection rates of PE before 34 gestation week $(\mathrm{GW})$ were $59 \%$ with $5 \%$ false positive rate and $75 \%$ with $10 \%$ false positive rate. ${ }^{9}$ For IUGR prediction, the sensitivity of first trimester uterine artery PI was $12 \%$ in the general population and $34 \%$ in the high-risk population. ${ }^{10}$ In comparison with other Doppler indices and biomarkers, the uterine artery PI at 22 GW still had better performance for severe PE prediction. However, its early predictive performance, which was the uterine artery PI value in first trimester (12 GW), remained unsatisfactory. ${ }^{11}$ In addition, the uterine artery, as the main afferent vessel of uteroplacental unit, is a partial reflect of real haemodynamic conditions within the placenta. Therefore, a non-invasive quantification of intervillous space vascularisation might be of great interest to obtain more valuable information about placental function.

Since 2004, it has been possible to quantify the placental and myometrium vascularisation by three-dimensional (3D) ultrasound angiography. As a very promising approach in clinic, this technique permits a direct evaluation of perfusion of an organ of interest. In a prospective observational multicentre study, Hafner et al showed that 3D Power Doppler angiography (3DPD), a new imaging tool to quantify the vascularisation within the uteroplacental unit, had promising predictive power to identify a PE-pregnancy already at 12 weeks. ${ }^{11}$

With the development of 3DPD angiography, it is possible to quantify the power Doppler signals within a volume or an organ of interest. This quantification is based on three typical indices: Vascularisation Index (VI), Flow Index (FI) and vascularisation-FI (VFI). VI (\%) is the ratio of colour-coded voxels (with moving blood) to all voxels in a given volume of interest (VOI). FI is the mean value of all colour-coded voxels in the vessels of the segmented volume expressed in arbitrary unit. VFI is the product of the latter indices. ${ }^{12} \mathrm{~A}$ high intraobserver and interobserver reproducibility and its potential interest for placental function study were reported by our team. ${ }^{13-15}$

The main limit of this technique is that the calculated 3DPD indices are dependent on different physiological parameters (such as body mass index (BMI), placenta location) and machine settings. This has been proved in vitro, and it is also considered as a main cause of heterogeneous previously published data. ${ }^{16-18}$ Our team also evaluated the impact of different machine settings on 3DPD indices in a sheep model.

Despite these limitations, the technique has provided interesting results: a significant drop of placental VI, FI and VFI were rapidly observed in pre-eclamptic patients during the first trimester, as well as during the second and the third trimesters. ${ }^{19}{ }^{20}$ In addition, the decrease of these 3DPD indices appeared earlier than anomalies of uterine artery PI. 3DPD indices showed a great superiority in terms of prediction of PE compared with the uterine artery PI at the end of the first trimester. ${ }^{21-23}$

The placental origin of IUGR was also evaluated by 3DPD. In a nitric oxide treated IUGR rabbit model, which was built by our team, the placental indices (VI, FI and VFI) were significantly reduced at 28 days of gestation. ${ }^{24}$ In human being, a reduction of placental VI and VFI was always observed in IUGR patients during second and third trimesters. ${ }^{25-27}$ A positive correlation between 3DPD indices and IUGR severity was also established. ${ }^{28}$ Recently, over a larger population (4325 pregnant women), the myometrial VI in the first trimester has shown its best performance for all types of PE prediction, and it was also as good as uterine artery PI in the second trimester for severe PE prediction. ${ }^{11}$ As shown above, the 3D Doppler measurements proved its value and great potential for placental origin disease prediction, especially the $\mathrm{PE}$ and IUGR.

The evaluation of the uteroplacental vasculature in the first trimester using 3DPD, a very recently developed approach, is a technique mastered in practice by only few teams. Our group has been a pioneer and has a recognised expertise in the field of 3DPD. Teams involved in this project have expertise in both the field of study of $\mathrm{PE}$ and in the management of prospective cohort of pregnant women.

Concomitant prospective and concurrent evaluation of all tools available for the prediction of both PE and IUGR in the general population has never been performed. All data will be combined to develop an integrated algorithm for the early prediction of PE and IUGR. We expect to improve our ability to predict the onset of both PE and IUGR, including late onset and mild PE. The availability of such predictive tools would have a major benefit for the prevention of PE, IUGR and their complications in the near future.

\section{METHODS AND ANALYSIS Objectives}

The primary objective is to determine the differences in 3DPD indices at the first trimester between pregnancies defined at their outcome as uncomplicated pregnancy, PE (mild and severe) and IUGR in nulliparous women.

The secondary objectives are: 
1. To evaluate and compare the predictive values of 3DPD, maternal characteristics, uterine artery Doppler (UAD) and current biomarkers (PAAP-A, PIGF) for PE and/or IUGR presence.

2. To evaluate 3DPD predictive performances stratified by:

- Machine settings (five different settings).

- Uteroplacental analysis (manual or automated).

- Maternal characteristics affecting the 3DPD signal: placental localisation and BMI.

- Personal indicators of individual risk for PE and IUGR: tobacco intoxication, BMI, chronic arterial hypertension, autoimmune or renal disease, haemostasis disorders, diabetes.

3. To evaluate intraobserver and interobserver variability for 3DPD analysis (random sample of 60 placenta).

4. To assess the predictive value of the markers for the occurrence of other pregnancy complications (neonatal mortality and morbidity, placental abruption, preterm delivery).

5. If we confirm the main hypothesis, we will develop an algorithm for the prediction of PE and/or IUGR presence, including 3DPD, UAD, biomarkers and maternal characteristics, using the prospective Standard Protocol Items: Recommendations for Interventional Trials (SPIRIT) cohort data using a 2/3 random sample (logistic regression model with stepwise variable selection). The model validation will be done in the $1 / 3$ random sample.

6. To constitute a biological collection of blood samples in order to determine diagnostic markers or severity predictive markers for PE and/or IUGR.

The objective of the ancillary study (SPIRIT-REPRO ie, SPIRIT-Reproducibility) is to evaluate interobserver and interdevice variability at the time of data acquisition.

\section{Trial design}

The SPIRIT protocol is a national, multicentre prospective cohort study concerning four French centres: Centre Hospitalier Régional Universitaire (CHRU) of Nancy, Paris-APHP Port-Royal, CHU of Strasbourg and BelfortHôpital Nord Franche-Comté.

The ancillary study SPIRIT-REPRO will be performed in the main centre (CHRU of Nancy).

\section{Study population}

Women will be informed and recruited during their first trimester consultation or first trimester ultrasonography screening (11-13+6GW), over a period of 36 months. All the nulliparous pregnant women who agree to participate in the study will be recruited, despite their medical history and previous pregnant examination results. The inclusion and exclusion criteria are reported in table 1. The study information and consent collection will be provided by the investigators (obstetricians or midwives) (online supplemental annex1-3).

\section{Doppler acquisitions and blood sampling}

The 3DPD and UAD acquisitions will be performed immediately after the first trimester ultrasound screening and followed by blood sampling $(20 \mathrm{~mL}$ in total) for biomarker analysis (PAPP-A and P1GF) and biological collection.

Investigators and midwives of each centre will be trained to use the ultrasound system (Voluson S8) in a standardised fashion, with standardised acquisition protocol and machine settings, to secure optimal reproducibility (table 2).

To determine an optimal 3DPD machine setting in a general population (secondary objective 2), several predetermined settings will be used for 3DPD acquisition.

\section{Image analysis}

VOI will be drawn using VOCAL software and three 3DPD indices will be automatically calculated: VI, FI, VFI (figure 1).

\section{Outcomes}

The primary endpoint is the occurrence of $\mathrm{PE}$ and IUGR and quantitative results of 3DPD: uteroplacental, myometrial and placental VIs.

$\mathrm{PE}$ is defined by an arterial hypertension $>140 / 90 \mathrm{~mm}$ $\mathrm{Hg}$ pre-existing or occurring after $20 \mathrm{GW}$ and disappearing before the end of sixth postpartum week associated with a proteinuria $>0.3 \mathrm{~g} / 24$ hours or existence of end organ damage.

IUGR is defined by a birth weight $<10$ th centile for the gestational age.

The secondary endpoints are:

1. Occurrence of PE and IUGR. 3DPD, PAPP-A and PIGF markers diagnostic performance will be assessed by

Table 1 Inclusion and exclusion criteria

\begin{tabular}{|c|c|}
\hline Inclusion criteria & Exclusion criteria \\
\hline $\begin{array}{l}\text { Singleton nulliparous pregnancy between } 11 \text { and } 13+6 \mathrm{GW} \text {, } \\
\text { Patient over } 18 \text { years old, } \\
\text { Mandatory enrolment in a social security plan, } \\
\text { Patient (or a third person, independent from the investigator } \\
\text { and the sponsor, in case of inability to read or write) having } \\
\text { signed an informed consent. }\end{array}$ & $\begin{array}{l}\text { Patients under a measure of legal protection, } \\
\text { Patient participating simultaneously to other interventional } \\
\text { research trial to test a pharmaceutical treatment and protocols } \\
\text { are not compatible, } \\
\text { Inability to understand and accept the protocol. }\end{array}$ \\
\hline
\end{tabular}

GW, gestation week. 
Table 2 Standardised protocol for 3DPD acquisition

General setting

\section{Power Doppler Mode}

No modification of acquisition window

No modification of gain (except for the setting 5)

the operator only has to select the corresponding setting

$\begin{array}{ll}\text { Setting 1 } & \text { PRF 0.9, Gain -7.2, WMF moy } \\ \text { Setting 2 } & \text { PRF 1,8, Gain -7.2, WMF moy } \\ \text { Setting 3 } & \text { PRF 0.9, Gain -7.2, WMF max } \\ \text { Setting 4 } & \text { PRF 0.9, Gain -7.2, WMF low } \\ \text { Setting 5 } & \text { PRF 0.9, Gain -7.2, WMF moy and } \\ & \begin{array}{l}\text { manually modification of gain to } \\ \text { sub-noise gain (increase gain to } \\ \text { artefact limits) }\end{array}\end{array}$

3DPD, three-dimensional power Doppler; PRF, pulse repetition frequency; WMF, wall motion filter.

calculating area under receiver operating characteristic (ROC) curve.

2. Occurrence of PE and IUGR in each subgroup (PE, IUGR and PE +IUGR) according to maternal characteristics (BMI and placental localisation) and machine settings (five different settings). The diagnostic performance of 3DPD in each subgroup of settings, maternal characteristics and placental localisation will be assessed by the calculation of the area under the ROC curve. This will be compared using statistics among all available markers tests.

3. Intraclass correlation coefficient for 3DPD indices (intraobserver and interobserver measurements).

4. Occurrence for neonatal mortality and morbidity.

Occurrence of a placental abruption.

Occurrence of preterm delivery defined by birth before $37 \mathrm{GW}$.

Occurrence of preterm delivery before 34 GW.
5. If we confirm the main hypothesis, we will develop an algorithm for the prediction of PE and IUGR, including 3DPD, UAD, biomarkers and maternal characteristics, using the prospective SPIRIT cohort data using a 2/3 random sample (logistic regression model with stepwise variable selection). The model validation will be done in the $1 / 3$ random sample.

6. Obtaining analysable blood samples for diagnostic markers and severity predictive markers of $\mathrm{PE}$ and IUGR study.

The endpoints of the ancillary study (SPIRIT-REPRO) are inter-observer reproducibility and inter-device reproducibility of the 3DPD measurements.

\section{Participant timeline}

The enrolment of women started in April 2018. The recruitment should be achieved by April 2021. The flow chart of patient participation is presented in figure 2 .

\section{Premature ending of patient participation}

Every person can stop participating in the research at any time and for whatever reason.

The investigator may temporarily or permanently interrupt a person's participation in the research for any reason that has an impact on her safety or that would best serve the interests of the person who is suitable for research.

In the event of a premature ending or in the event of the withdrawal of consent, the withdrawal shall not affect the activities carried out and the use of data obtained on the basis of informed consent before it has been withdrawn, unless the person indicates in writing that she objects to their use.

When a patient withdraws her consent, the data acquired before the withdrawal will be available for the statistical analysis unless refusal from the patient. A procedure to replace patients ending prematurely their participation in the study will be implemented if more than $5 \%$ of premature endings are observed.

Figure 1 Three dimensional power Doppler (3DPD) analysis. FI, Flow Index; VFI, Vascularisation-FI; VI, Vascularisation Index. 


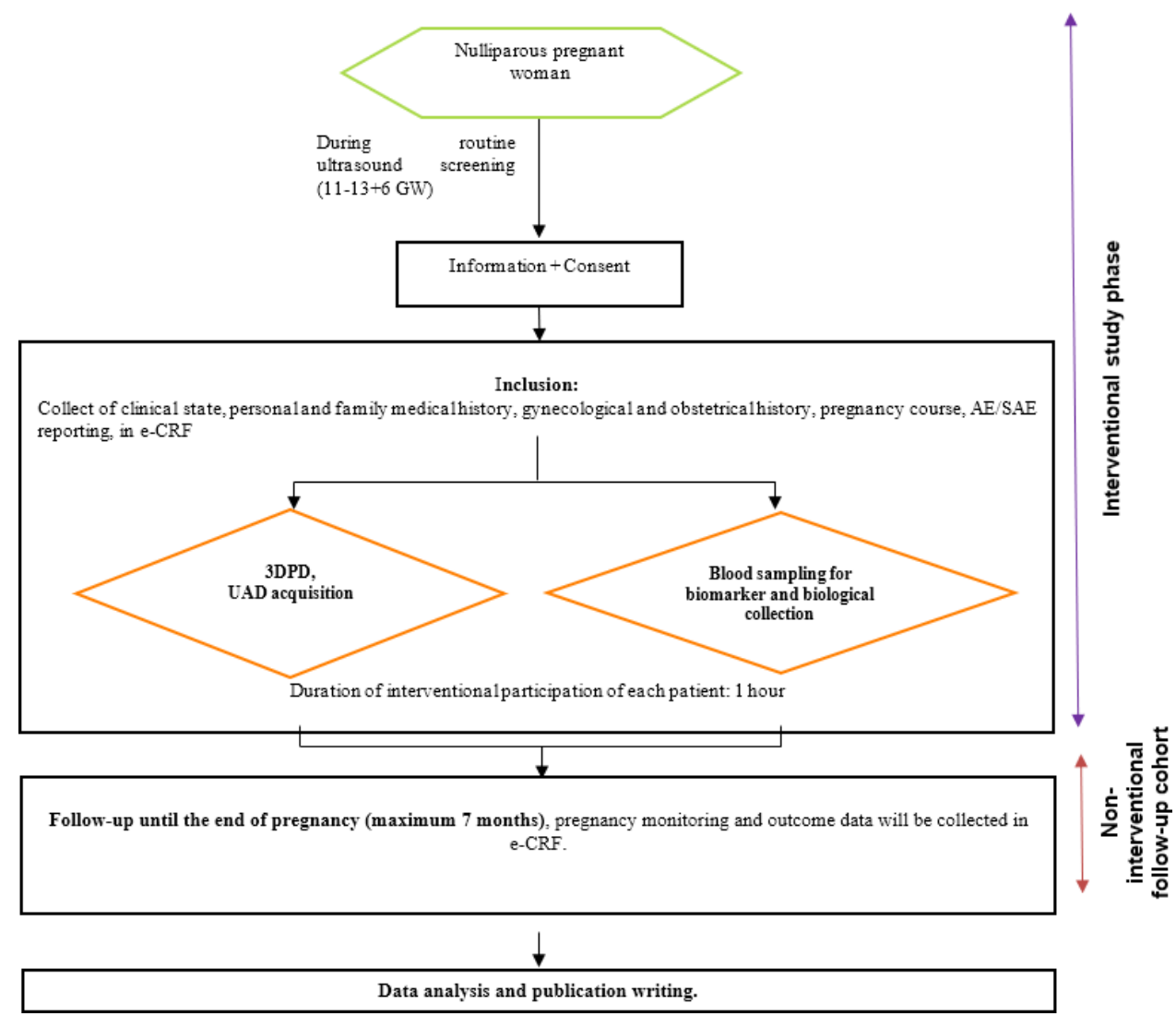

Figure 2 Flow chart of patient participation. AE, adverse event; 3DPD, three-dimensional power Doppler; eCRF, electronic case report form; GW, gestation week; SAE, serious AE; UAD, uterine artery Doppler.

\section{Follow-up}

No specific follow-up is organised for patients ending, prematurely or not, their participation in the study. After childbirth, the pregnancy monitoring and outcome data (medical routine follow-up) will be collected for further analysis. Only routine data will be collected, routine pregnancy monitoring will not be changed. The data will be collected from the patient's medical file. Pregnancy course and outcome of all participants will be recorded on electronic case report forms (eCRFs) and added to the database.

\section{Ancillary study (SPIRIT-REPRO)}

For the ancillary study, 3DPD acquisitions will be repeated in one centre (CHRU of Nancy). A second operator (doctor or midwife) will perform the same 3DPD series on the same device (Voluson S8). Also, the same series will be performed by the first operator using another device (Voluson E10).

This ancillary study provides the inclusion of 30 women at the Nancy CHRU to study the interobserver and interdevice variability of the 3DPD measurements.

\section{Sample size consideration}

The sample size is based on mean comparison for IUGR and PE compared with uncomplicated pregnancy and has been estimated using MedCalc Software. In Hafner et al study, the mean 3DPD index measured was about $35.6 \pm 12.9$ in the uncomplicated group, $31.4 \pm 13.9$ in the group with IUGR and $19.4 \pm 11.35$ for PE group. So, to show a significant difference between uncomplicated pregnancy and IUGR in 3DPD measurements, with a $100 / 10$ ratio a specified power of $90 \%$ and a type I error of $1 \%$ (to control multiple comparisons); we need to include in statistical analysis 1961 women. Considering that in the obstetrical field the expected lost to follow-up women is relatively low, around $10 \%$, we should include 2200 women in the SPIRIT cohort. As expected differences between uncomplicated pregnancy and PE are bigger than those for IUGR, the sample size will also allow to show a significant difference between uncomplicated pregnancy and PE. The ancillary study is a feasibility study, and we decided to include 30 women.

\section{Data collection and management}

The collection of personal history, biomarkers, 3DPD and UAD acquisitions will be performed at the time of inclusion. 3DPD and UAD acquisitions will be carried out by specially trained midwives, including the collection and handling of blood samples for biomarkers assays and biological collection. The quantitative results of the various tests will be collected and will remain blinded to the investigators and pregnant women. Data for this study will be recorded on an eCRF and are presented in table 3 .

The data from all women will be centrally stored in Nancy. Data management will be carried out by Nancy CIC-IT (INSERM CIC 1433). The conditions for data 
Table 3 Data collected

$\begin{array}{ll}\text { Maternal characteristic } & \text { Body mass index; smoking status; conception mode } \\ \text { Medical history } & \text { Diabetes; arterial hypertension; thrombophilia; current treatments } \\ \text { 3DPD data } & \text { Pulsatility index in uterine artery; } \\ & \text { Placental volume; myometrium volume } \\ & \text { 3DPD indices for placental and myometrium (VI, FI, VFI) for different the five machine settings } \\ \text { Biomarkers } & \text { PAAP-A (mUI/L); PIGF (pg/mL) } \\ \text { Pregnancy follow-up } & \text { Ultrasound (estimation of fetal weight and Doppler resistance index) at different gestational age } \\ \text { Pregnancy outcomes } & \text { No of hospitalisations } \\ & \text { Occurrence of pre-eclampsia or IUGR } \\ & \text { Gestational age and mode of delivery } \\ & \text { Mode of labour induction } \\ \text { Neonatal outcomes } & \text { Neonatal weight ; Apgar score } \\ & \text { Umbilical artery pH; base excess; lactate } \\ \text { Resuscitation; Admission in neonatal intensive care unit }\end{array}$

3DPD, three-dimensional power Doppler; FI, Flow Index; IUGR, intrauterine growth restriction; VFI, Vascularisation-FI; VI, Vascularisation Index.

transfer of all or part of the study database are decided by the study sponsor and are the subject of a written contract. The data images will be transferred to Nancy CIC-IT and stored after verification, in the ArchiMed database declared to the French authority (CNIL declaration number: 1410005). This study and the data collected fall within the scope of Reference Methodology MR001.

After data collection, women will be allocated to a group including:

- Uncomplicated pregnancy.

- Complicated pregnancy.

- By PE

- By IUGR

- By PE and IUGR.

\section{Statistical analysis}

Statistical analysis will be performed by Nancy CIC. All analyses will be performed with $\mathrm{R}$ software. Continuous variables will be expressed as mean $\pm \mathrm{SD}$, categorical variables as frequencies (percentages). A $\mathrm{p}<0.05$ will be considered to be significant. A Bonferroni correction will be performed for multiple comparisons.

The aim of the trial is to determine differences in 3DPD indices between uncomplicated pregnancy, PE (mild and severe) and IUGR in nulliparous women. For each subgroup mean values in 3DPD indices will be computed and compared using a pairwise $t$ test with a Bonferroni correction $p$ value adjustment.

Sensitivity, specificity, and their exact $95 \%$ CIs, positive predictive value (PPV), negative predictive value (NPV) and accuracy of 3DPD, maternal characteristics, UAD and current biomarkers will be calculated using the binomial distribution. These values will be compared with each other. The interobserver and intraobserver reproducibility for 3DPD analysis will be evaluated by calculating an intraclass correlation coefficient (and its 95\% CI), complemented by a Bland-Altman graph. For ancillary study, the interobserver and intermachine reproducibility will be evaluated by calculating, on a sample of 60 women, intraclass correlation coefficients (and its 95\% CI), complemented by Bland-Altman graphs. After quantification of uteroplacental vascularisation, an algorithm for the early prediction of PE and/or IUGR based on 3DPD will be developed using the cohort of 2200 participants. The data will randomly split into two parts: $2 / 3$ to develop the model to predict PE and/or IUGR occurrence (train data) and the remaining $1 / 3$ to measure its performance (test data). Diagnostic performance to predict PE and/or IUGR occurrence will be assessed by calculating univariate binomial regression and its corresponding ROC curve for each marker (such as biomarkers, maternal characteristics, 3DPD values) on the train data. Variables with a $<15 \%$ in this univariate analysis were considered further. On the basis of the ROC analysis, the sensitivity, specificity, PPV, NPV, and accuracy of PE and/or IUGR presence will be calculated. The final model will be selected in order to maximise sensitivity. Finally, sensitivity and specificity will be calculated on the test data. Furthermore, we will evaluate whether the predictive capacity of this algorithm is better than the biomarkers, and then be improved by adding additional biomarkers, $\mathrm{UAD}$ and maternal characteristics.

\section{Patient and public involvement}

Patients and public were not involved.

\section{ETHICS AND DISSEMINATION}

The study was approved by the French Ethics Committee, the Comité de Protection des Personnes (CPP) SUD MEDITERRANEE IV on 13 February 2018 with reference number 171203 . The results of this study will be published 
in a peer-reviewed journal and will be presented at relevant conferences.

\section{Quality control}

Right of access to data and source documents

The CHRU is responsible for obtaining the agreement of all parties involved in the study so as to guarantee direct access to all study sites, source data, source documents, and reports so that the sponsor may control data quality and perform an audit.

Investigators will make available the documents and individual data strictly required for monitoring, quality control and audit of the biomedical study to persons having access to these, in accordance with the statutory and regulatory provisions in place (articles L.1121-3 and R.5121-13 of the French Public Health Code).

Any original document or object that allows the existence or accuracy of a data point or information recorded during the study to be proved is defined as a source document.

In accordance with the statutory provisions in place (articles L.1121-3 and R.5121-13 of the French Public Health Code) the persons having direct access to source data will take every precaution required to ensure the confidentiality of information relating to investigational medicinal products, studies, participants, notably concerning the identity of these, as well as the results obtained. These persons, as the investigators themselves, are subject to professional confidentiality.

During the study, or at its conclusion, data collected regarding participants that is sent to the sponsor by the investigators (or all other specialists involved) will be coded by the inclusion number of the patient in the study. At no point should the names of participants or their addresses appear unencrypted. The presentation of the data processing results can not in any case allow the direct or indirect identification of persons lending themselves to research.

The sponsor will ensure that each study participant has given her written consent for access to her personal data that is strictly required for study quality control.

The data may be transmitted to the companies in the group to which the promoter belongs and to its contractual partners in a form which should not permit the direct or indirect identification of persons lending themselves to research.

\section{Study monitoring}

The monitoring visits (implementation, follow-up and closure) will be performed by the promoting cell of Nancy Délégation à la Recherche Clinique et à l'Innovation - CHRU Nancy.

A Clinical Research Associate will travel regularly to each centre to perform the quality control of the study.

Depending on monitoring reports and deviations observed, the promoter reserves the right to modify the level of monitoring initially planned.

\section{Potential risks related to the study}

There are no risks for the patient and for the fetus. The only constraint for the patients is the addition of maximum $10 \mathrm{~min}$ long ultrasound and a $20 \mathrm{~mL}$ maternal blood sample during first trimester routine consultation.

For pregnant women participating also in the ancillary study (SPIRIT-REPRO), the only constraint for the patients is the addition of maximum $20 \mathrm{~min}$ long ultrasound.

The 3DPD medical device is CE marked and is used routinely in the clinic.

\section{Ethical permission}

The sponsor and investigators undertake to carry out this research in accordance with the recommendations of the Helsinki Declaration and its revisions, the European Regulation (EU) no 536/2014 from the European Parliament about clinical trials of medicines for human use, repealing European Directive 2001/20/CE, the no 2004806 law of 9 August 2004 about public health policy, the no 2004-800 law of 6 August 2004 about bioethics, the No 78-17 law of 6 January 1978 relating to data processing, files and freedoms, the $\mathrm{n}^{\circ}$ 2012-300 law of 5 March 2012 about research involving the human person, the 2016-41 law of 26 January 2016 of modernisation of our healthcare system and 2016-800 ordinance of 16 June 2016 relating to researches involving the human person and their implementing decrees.

They undertake to comply with all laws and regulations that may apply to research.

The investigators undertake to respect the protocol in all respects especially with regard to obtaining consent and the notification and follow-up of serious adverse events.

\section{Protocol Amendment}

Requests of authorisation and/or opinion about substantial amendments will be addressed by sponsor to regulatory institutions.

By signing this protocol, the investigator commits to submit to the Direction of Research and Innovation the substantial amendment project and wait for authorisation and/or opinion of regulatory institutions prior to the application of amendment.

\section{Final research report}

The final report of the research will be written collaboratively by the coordinator and the biostatistician mandated for this search. This report will be submitted to each of the investigators for review. Once a consensus has been reached, the final version must be endorsed with the signature of each of the investigators and sent to the sponsor as early as possible after the effective end of the research. A report prepared according to the reference plan of the competent authority must be forwarded to the competent authority and the CPP within a year after the end of the research, understood as being the last follow-up visit of 
last enrolled subject. This period is abrogated to 90 days in case of premature termination of the research.

\section{DISCUSSION}

The main objective of this study is to confirm the interest of 3DPD for PE and IUGR first-trimester screening.

This study was designed before the publication of the ASPRE trial results. ${ }^{29}$ In their study, the observed rate of $\mathrm{PE}$ after screening was lower than expected. These results enhanced the interest of our study because there is still no effective screening technique for prediction of $\mathrm{PE}$ and IUGR in the first trimester.

Our study is, to our knowledge, the only and the first large cohort study with a prospective design that will be able to conclude about 3DPD performance in PE/IUGR screening in the first-trimester. In case of demonstrated interest, the routine clinical application could be soon.

The main question with 3DPD, currently unresolved, is the impact of machine settings and intra and inter observer variability for acquisition and analysis. This point will also be addressed in our study and that is one of its strengths.

The strategy used for the study design was not just to test one new biomarker vs another. In order to achieve a good prediction tool, we think it is necessary to consider the respective potential advantages of the different approaches. Therefore, all markers already tested in other studies will be collected. First, these data will be compared with 3DPD, then, it will be associated with 3DPD markers to propose a decision algorithm.

The evaluation of placental development by exploiting imaging technologies, such as 2D colour Doppler and 3DPD represents the state-of-the-art in the obstetrics research field, and such an approach has not yet reached the clinic. Inclusion of these non-invasive imaging techniques in a PE/IUGR risk assessment procedure will give a considerable benefit in terms of regulatory approval of patient stratification methods and clinical implementation of these techniques. The expected benefit for the pregnant women or their children is important, which includes better monitoring for pathological pregnant patients and reduction of mother and child morbidity and mortality.

Furthermore, the poor predictive value of nowadays available tests is a great limitation for the exploration of new preventive treatments. Indeed, as the rate of PE remains very low in a screened 'high-risk' group $(4.6 \%$ in the ASPRE study), the number of pregnant women to be included in a prospective randomised trial to validate the efficiency of a treatment versus placebo is too high.

Contributors $\mathrm{OM}, \mathrm{GH}, \mathrm{MB}$ contributed to the conception and designof the study, $\mathrm{OM}$ and $\mathrm{CBe}$ are the coordinating investigators; $\mathrm{CBa}$ is the study project manager; $\mathrm{CBe}$ wrote the manuscript; $\mathrm{CBe}$ will carry out recruitment, ultrasound acquisition and will collect the data; $\mathrm{MB}$ is supervising data processing; $\mathrm{GH}$ is in charge of statistical analysis and all authors reviewed and contributed to the manuscript. All authors have read, approved the paper and meet the criteria for authorship as established by the International Committee of Medical Journals Editors.
Funding This study is supported by a grant from the French Ministry of Health (2016) with the number PHRCN-16-0567.

Competing interests None declared.

Patient and public involvement Patients and public were involved in the study (Annex 1-3).

Patient consent for publication Not required.

Ethics approval The French Ethics Committee (Comite de Protection des Personnes, CPP) approved this study (Reference number 1712 03).

Provenance and peer review Not commissioned; externally peer reviewed.

Open access This is an open access article distributed in accordance with the Creative Commons Attribution Non Commercial (CC BY-NC 4.0) license, which permits others to distribute, remix, adapt, build upon this work non-commercially, and license their derivative works on different terms, provided the original work is properly cited, appropriate credit is given, any changes made indicated, and the use is non-commercial. See: http://creativecommons.org/licenses/by-nc/4.0/.

ORCID iD

Charline Bertholdt http://orcid.org/0000-0001-9297-5363

\section{REFERENCES}

1 Kaufmann P, Black S, Huppertz B. Endovascular trophoblast invasion: implications for the pathogenesis of intrauterine growth retardation and preeclampsia. Biol Reprod 2003;69:1-7.

2 Martin CB, Ramsey EM, Donner MW. The fetal placental circulation in rhesus monkeys demonstrated by radioangiography. Am J Obstet Gynecol 1966;95:943-7.

3 Donner MW, Ramsey EM. Radioangiographic studies on the dynamics of the blood circulation in the maternal placenta. (Experimental studies on the rhesus monkey)]. Fortschr Geb Rontgenstr Nuklearmed. juin 1966;104:796-808.

4 Jaffe R, Woods JR. Doppler velocimetry of intraplacental fetal vessels in the second trimester: improving the prediction of pregnancy complications in high-risk patients. Ultrasound Obstet Gynecol 1996;8:262-6.

5 Jaffe R, Jauniaux E, Hustin J. Maternal circulation in the firsttrimester human placenta--myth or reality? Am J Obstet Gynecol 1997;176:695-705.

6 Jurkovic D, Jauniaux E, Kurjak A, et al. Transvaginal color Doppler assessment of the uteroplacental circulation in early pregnancy. Obstet Gynecol 1991;77:365-9.

7 Schaaps J-P, Tsatsaris V, Goffin F, et al. Shunting the intervillous space: new concepts in human uteroplacental vascularization. Am J Obstet Gynecol 2005;192:323-32.

8 Bertholdt C, Eszto M-L, Tournier M, et al. Assessment of uteroplacental vascularisation in early first-trimester pregnancy with contrast-enhanced ultrasound and 3D power Doppler angiography: protocol for a prospective, cross-sectional, multicentre and nonrandomised open study ("HOPE Study"). BMJ Open 2019;9:e030353.

9 Poon LC, Nicolaides KH. Early prediction of preeclampsia. Obstet Gynecol Int 2014;2014:1-11.

10 Cnossen JS, Morris RK, ter Riet G, et al. Use of uterine artery Doppler ultrasonography to predict pre-eclampsia and intrauterine growth restriction: a systematic review and bivariable meta-analysis. CMAJ 2008;178:701-11.

11 Hafner E, Metzenbauer M, Stümpflen I, et al. Measurement of placental bed vascularization in the first trimester, using 3D-powerDoppler, for the detection of pregnancies at-risk for fetal and maternal complications. Placenta 2013;34:892-8.

12 Schulten-Wijman MJNC, Struijk PC, Brezinka C, et al. Evaluation of volume vascularization index and flow index: a phantom study. Ultrasound Obstet Gynecol 2008;32:560-4.

13 Morel O, Grangé G, Fresson J, et al. Vascularization of the placenta and the sub-placental myometrium: feasibility and reproducibility of a three-dimensional power Doppler ultrasound quantification technique. A pilot study. J Matern Fetal Neonatal Med 2011;24:284-90.

14 Cabezas López E, Martínez-Payo C, Engels Calvo V, et al. Reproducibility of first trimester three-dimensional placenta measurements. Eur J Obstet Gynecol Reprod Biol 2016;201:156-60.

15 Reijnders IF, Mulders AGMGJ, Koster MPH, et al. New imaging markers for preconceptional and first-trimester utero-placental vascularization. Placenta 2018;61:96-102.

16 Raine-Fenning NJ, Nordin NM, Ramnarine KV, et al. Evaluation of the effect of machine settings on quantitative three-dimensional 
power Doppler angiography: an in-vitro flow phantom experiment. Ultrasound Obstet Gynecol 2008;32:551-9.

17 Raine-Fenning NJ, Nordin NM, Ramnarine KV, et al. Determining the relationship between three-dimensional power Doppler data and true blood flow characteristics: an in-vitro flow phantom experiment. Ultrasound Obstet Gynecol 2008;32:540-50.

18 Chen CY, Chang HT, Chen CP, et al. First trimester placental vascular indices and volume by three-dimensional ultrasound in pre-gravid overweight women. Placenta 2019;80:12-17.

19 Dar Pe'er, Gebb J, Reimers L, et al. First-Trimester 3-dimensional power Doppler of the uteroplacental circulation space: a potential screening method for preeclampsia. Am J Obstet Gynecol 2010;203:238.e1-238.e7.

20 Costa J, Rice H, Cardwell C, et al. An assessment of vascularity and flow intensity of the placenta in normal pregnancy and pre-eclampsia using three-dimensional ultrasound. J Matern Fetal Neonatal Med 2010;23:894-9.

21 Hafner E, Metzenbauer M, Stümpflen I, et al. First trimester placental and myometrial blood perfusion measured by 3D power Doppler in normal and unfavourable outcome pregnancies. Placenta 2010;31:756-63.

22 Demers S, Girard M, Roberge S, et al. First-Trimester placental and myometrial blood perfusion measured by three-dimensional power Doppler in preeclampsia. Am J Perinatol 2015;32:920-6.
23 González-González NL, González Dávila E, Padrón E, et al. Value of placental volume and vascular flow indices as predictors of early and late preeclampsia at first trimester. Fetal Diagn Ther 2018;44:256-63.

24 Lecarpentier E, Morel O, Tarrade A, et al. Quantification of utero-placental vascularization in a rabbit model of IUGR with three-dimensional power Doppler angiography. Placenta 2012;33:769-75

25 Guiot C, Gaglioti P, Oberto M, et al. Is three-dimensional power Doppler ultrasound useful in the assessment of placental perfusion in normal and growth-restricted pregnancies? Ultrasound Obstet Gynecol 2008;31:171-6.

26 Noguchi J, Hata K, Tanaka H, et al. Placental vascular sonobiopsy using three-dimensional power Doppler ultrasound in normal and growth restricted fetuses. Placenta 2009;30:391-7.

27 Pomorski M, Zimmer M, Florjanski J, et al. Comparative analysis of placental vasculature and placental volume in normal and IUGR pregnancies with the use of three-dimensional power Doppler. Arch Gynecol Obstet 2012;285:331-7.

28 Luria O, Barnea O, Shalev J, et al. Two-Dimensional and threedimensional Doppler assessment of fetal growth restriction with different severity and onset. Prenat Diagn 2012;32:1174-80.

29 Rolnik DL, Wright D, Poon LC, et al. Aspirin versus placebo in pregnancies at high risk for preterm preeclampsia. N Engl J Med 2017;377:613-22. 